Estudios Románicos, Volumen 28, 2019, pp. 99-113

ISSN: 0210-491

eISSN: 1989-614X

DOI: https://doi.org/10.6018/ER/372141

\title{
DACIA MARAINI Y EL GÉNERO EPISTOLAR: CHIARA D'ASSISI. ELOGIO DELLA DISOBBEDIENZA
}

(Dacia Maraini and the epistolary genre in Chiara d'Assisi. Elogio della Disobbedienza)

\section{Almudena Miralles Guardiola* Universidad de Murcia}

\begin{abstract}
The epistolary genre has profusely been chosen by Dacia Maraini as an appealing and effective resource when it comes to transmit the message to the reader. The present paper suggests a tour through the several works in which the Italian writer has opted for this format and carries out an analysis of Mariani's intention when choosing the epistolary exchange in one of his most recent novels: Chiara d'Assisi. Elogio della Disobbedienza.
\end{abstract}

Keywords: Epistolary genre; Maraini; Chiara d'Assisi; Happy body.

Resumen: El género epistolar ha sido muy frecuentemente elegido por Dacia Maraini como un recurso atractivo y eficaz a la hora de hacer llegar el mensaje al lector. El presente trabajo propone un recorrido por las distintas obras en las que la escritora italiana se ha decantado por este formato y lleva a cabo un análisis de la intención de Maraini al elegir el intercambio epistolar en una de sus novelas más recientes: Chiara d'Assisi. Elogio della disobbedienza.

Palabras clave: Género epistolar; Maraini; Chiara d'Assisi; Corpo felice.

Este trabajo muestra la elección y el tratamiento del género epistolar en la obra de Dacia Maraini en relación con las estrategias narrativas de esta autora y la intención de hacer llegar su mensaje literario de una forma directa, convincente y que implica a los lectores en el relato. En la obra de Maraini, el género epistolar se ha revelado como el

*Dirección para correspondencia: Almudena Miralles Guardiola. Plaza Santa Ana 2, 30008 Murcia (almudena. miralles@um.es) 
recurso más efectivo en el momento de trasmitir la personalidad y las vivencias de los personajes, al conseguir despertar con facilidad la empatía en el lector. Entre los temas que han interesado a la autora desde el comienzo de su actividad literaria, destaca principalmente su preocupación por la situación de la mujer en la sociedad. Debido a este hecho, la narrativa de Maraini se encuentra plagada de relatos de experiencias de mujeres desfavorecidas por su condición, en distintos contextos espacio-temporales y bajo multitud de circunstancias que son reflejo de la gran desigualdad entre los sexos que ha existido y existe en la actualidad: son mujeres violadas, maltratadas por los hombres de su entorno y marginadas sociales. Es el caso, por ejemplo, de las protagonistas de las novelas Memorie di una ladra (1972), Isolina, la donna tagliata a pezzi (1980) o La lunga vita di Marianna Ucrìa (1990). De este modo, una de las funciones que la propia autora atribuye a su narrativa es la de dar visibilidad y denunciar la realidad a la que se enfrentan las mujeres tan solo por el hecho de serlo; así como de recuperar a figuras femeninas olvidadas o desprestigiadas por el canon y que sin embargo tuvieron una gran relevancia en su contexto, como se verá en Chiara d'Assisi. Elogio della disobbedienza (2013), la novela que analizamos en este artículo.

El género epistolar en la época contemporánea no ha sido un recurso demasiado explotado en la literatura italiana, tal y como resalta Mercedes Rodríguez Fierro cuando afirma que:

La literatura italiana, al igual que la española, y frente a las literaturas inglesa y francesa, no abunda en desarrollos del género más cercanos a la contemporaneidad, más allá de los casos de Storia di una capinera (1871) de G. Verga o las Lettere di una novizia (1941) de G. Piovene. (Rodríguez Fierro 1997: 212)

Este hecho contrasta con la gran presencia del género en la literatura tradicional femenina desde el siglo XVII, no sólo en Francia con figuras como Madame de Rambouillet o Marie de Rabutin-Chantal, marquesa de Sévigné, sino también en Portugal y España con Sor Mariana Alcoforado y Sor Juana Inés de la Cruz. Posteriormente se observa una progresiva restricción del recurso epistolar al ámbito literario francés e inglés. En el citado artículo, Rodríguez Fierro destaca la figura de Natalia Ginzburg como la gran cultivadora del género en Italia desde los años 70 del siglo pasado, con Caro Michele (1973) y La città e la casa (1984) (Rodríguez Fierro 1997: 212). Es durante la década siguiente cuando Dacia Maraini inicia su andadura en este sentido, y desde entonces existen numerosos casos en los que la autora recurre a la correspondencia entre personajes para trasmitir en primera persona cómo viven en sus circunstancias y cómo se sienten ante los acontecimientos que les rodean. Esto ocurre en Lettere a Marina, novela publicada en 1981 e inédita en castellano, en la que Bianca se dirige por escrito a su amiga Marina sin esperar respuesta, con el único objetivo de expresar sus sentimientos y recuerdos. Esta novela encuentra su contexto en la realidad social y política de la Italia de la década de los 80 , cuando se consolida la gran variedad de tendencias de la segunda ola del feminismo surgidas en este país durante la década anterior. Dacia Maraini elige como protagonista a Bianca, una mujer que escribe abiertamente sobre 
su relación amorosa con Marina, hecho que en el momento de la publicación de la obra todavía es visto como trasgresor. La propia autora afirmaba años más tarde que eligió contar esa historia porque se sentía comprometida con los problemas relacionados con los roles, la sexualidad y la relación entre los géneros, ya que considera que afectan de lleno a las mujeres porque su situación en la sociedad pasa a través de eso. Según Paola Susana Solorza, en Lettere a Marina:

El formato epistolar funciona como un tipo de escritura que permite la confesión y la reflexión de la narradora sobre los acontecimientos que han formado parte de su vida, como un momento de auto-análisis que Bianca se concede, bajo la forma de un diálogo con una interlocutora ausente. (Solorza 2015:138)

Es decir, Solorza estaría resaltando la relación de proximidad e incluso intimidad que Maraini consigue establecer entre la protagonista de la novela y los lectores mediante la elección de esta estructura. En el mismo sentido se manifiesta Loreta de Stasio, quien destaca esta novela de Dacia Maraini ${ }^{1}$ y afirma que «su misma forma permite apretar en un tú cargado de afecto todas las historias de nuestro yo» (Arriaga 2006: 137). Este es el efecto que consigue la autora a través del recurso de la comunicación por carta, que el lector haga suyas las experiencias de los personajes que escriben y experimente de la forma más íntima unos sentimientos que pueden serle más o menos cercanos. Desde este punto de partida de su actividad novelística, Dacia Maraini va a acudir al recurso de las cartas en muchas de sus novelas, siempre persiguiendo el efecto mencionado. Es el caso, por ejemplo, de La lunga vita di Marianna Ucrì (1990), en la que la protagonista se comunica por escrito con el resto de personajes, y de Un clandestino a bordo, ensayo escrito por Maraini en 1996 y en el que reflexiona sobre la maternidad, el aborto y las cuestiones controvertidas que existen alrededor de estos temas. La autora abre el ensayo con una carta dirigida a su amigo Enzo Siciliano, en la que hace referencias a su propia experiencia de la pérdida de su hijo poco antes de que naciese. Al año siguiente Maraini publica la novela Dolce per sé, en la que de nuevo va a hacer uso de las cartas para que se establezca una relación más íntima entre las dos protagonistas, Vera y la sobrina de su pareja, una vez que la relación amorosa que mantenía Vera se termina. Para entonces, el vínculo que se ha establecido entre las dos es tan estrecho que Vera siente la necesidad de escribir a la niña Flavia y contarle sus experiencias de vida plasmándolas en papel.

Durante las dos décadas sucesivas y hasta el día de hoy, la actividad narrativa de Dacia Maraini ha continuado imparable, así como la presencia de epistolarios en sus relatos. Los encontramos por ejemplo en Il treno dell'ultima notte, obra publicada en 2008 , en la que la autora vuelve a reflejar los sentimientos de los personajes, que en este caso protagonizan una historia de amor cuando son niños, en las cartas que intercambian durante ese tiempo. Amara es una periodista italiana que, aprovechando un encargo de trabajo, viaja hacia Centroeuropa en los años 50 y lleva consigo las cartas

1 En su capítulo «El teatro y la escritura femenina contemporánea: Recorrido, analogías y contrastes de dos dramaturgas italianas» incluido en Desde Andalucía: mujeres del Mediterráneo (Arriaga, 2006). 
que Emanuele le envió, con la esperanza de poder averiguar qué fue de él desde que se marchó con sus padres a Viena bajo la sombra del nazismo. En su última novela, Corpo felice. Storia di donne, rivoluzioni e un figlio che se ne va (2018), Maraini recurre de nuevo al formato epistolar y en una entrevista se refiere a la obra como una «lunga lettera» (Maraini en Vanity Fair, 2019) dirigida a su hijo perdido poco antes de nacer, un diálogo que establece con él imaginando que llegó a nacer y crecer. Pero es una novela un poco anterior y todavía inédita en castellano, Chiara d'Assisi. Elogio della disobbedien$z a$ (2013), de la que vamos a extraer el epistolario para analizar el estilo y la intención de la autora al elegir este formato. Es preciso destacar en este punto la especial atención que Maraini ha prestado en su obra a la literatura desarrollada por monjas, dado que las considera fundamentales para entender la historia de la literatura femenina y el desarrollo del protofeminismo. En este sentido, es indispensable mencionar una figura que impresionó a Maraini, la de santa Catalina de Siena, sobre la que escribió el texto teatral I digiuni di Caterina da Siena (2003). En una entrevista ese mismo año, la autora afirmaba:

Non sono cattolica. Ho cercato solo di laicizzare una figura dalla profonda spiritualità che teneva una corrispondenza con tutti i papi d' Europa e che ha condotto la sua esistenza con un amore militante. Per molte donne del passato il rapporto mistico con Dio era un segno di libertà, un accesso al di là dell' istituzione della Chiesa (Maraini 2003: 1)

Lo mismo sintió la escritora cuando descubrió a santa Clara de Asís, eje central de la novela que analizamos, una mujer medieval que desobedeció las normas impuestas a las mujeres de su clase en su tiempo, renunció a todas sus comodidades y huyó en busca de su independencia y libertad en sus votos y en la espiritualidad.

Chiara d'Assisi. Elogio della disobbedienza presenta una estructura dividida en dos partes: una primera parte en forma de novela epistolar, una segunda parte que se intercala en la primera y que aparece como una mezcla entre novela histórica y diario personal y el final en que la autora recupera el formato epistolar para concluir la narración. De este modo encontramos una apertura de la obra como novela epistolar y un cierre con el mismo diseño. En la parte que abre la novela comienza el intercambio de correspondencia entre la propia Dacia Maraini y una estudiante siciliana llamada Clara Mandalà, quien plantea la petición a la autora de que investigue y escriba sobre la figura de Santa Clara de Asís. Los motivos que llevan a la joven a dirigirse a Maraini para proponerle esta tarea los va exponiendo progresivamente en sus cartas, en respuesta a la contrariedad y las dudas que la escritora le trasmite en las suyas. Así, la relación que se establece entre ellas en un primer momento es de recelo por parte de Maraini, que no llega a entender las razones que han llevado a Clara Mandalà a buscarla para empujarla a escribir sobre Santa Clara. Esto es en un primer momento, veremos cómo evoluciona el tono y la relación entre las dos a medida que avanza el intercambio de correspondencia. En una de sus primeras cartas, la joven parece convencida de que existe una conexión entre su vida y la vida de la santa a través de los siglos y del nombre que comparten. 
Sono anni che mi chiedo chi sono, perché sinceramente non lo so. Per questo ho cominciato dal mio nome, sperando che mi aiutasse a capire. Qualche mese fa ho preso il treno e sono andata ad Assisi. Mentre viaggiavo ho letto un libricino sulla santa che ho trovato nella biblioteca del mio piccolo paese [...]. La città di Assisi mi ha riempita di meraviglia: forse perché l'ho vista subito con gli occhi del libro che sto leggendo, overo attraverso le ricostruzioni virtuali di uno storico del Medioevo [...]. Ma qui mi fermo perché non vorrei annoiarla. Vorrei che mi rispondesse. Lei sola mi può aiutare a capire. Io non capisco.

Con molta fiducia, Chiara Mandalà (Maraini 2013:10-12)

En cambio, Maraini le contesta que no comprende por qué se dirige a ella para llevar a cabo la investigación sobre la vida de la figura histórica y le contesta con aparente desgana respecto a la propuesta que, debido al interés que la joven muestra en Clara de Asís, la considera capaz de llevar a cabo las indagaciones por sí misma.

\section{Cara Chiara,}

Lei è proprio una strana creatura. Mi sta tirando per la manica in una vicenda che mi interessa poco. Perché non la scrive lei la storia di santa Chiara? Sono sicura che lo farebbe benissimo. Un caro saluto, D. M. (Maraini 2013:13)

Esta breve carta que Maraini envía en respuesta a una larga exposición que la estudiante le hace llegar previamente, en la que cuenta parte de sus circunstancias de vida y describe su visita a la ciudad de Asís y lo que este viaje despierta en ella, hace evidente para el lector que la escritora no tiene ningún interés en prestar atención a la sugerencia de la joven, y que sus palabras no han conseguido tampoco despertar la curiosidad en Maraini por descubrir a santa Clara. En cambio, este aparente rechazo de la autora a Clara Mandalà se revela como un efectivo recurso para que en el lector se empiece a despertar la curiosidad sobre la figura de la santa, el contexto medieval en el que vivió, y la relación que puede encontrar una estudiante del siglo XXI entre sus propias vivencias como mujer en la Italia actual con las experiencias de santa Clara como mujer en la Italia del siglo XIII. Se trataría así de un empleo del género epistolar en apariencia casual y sin una intención profunda, dado que las primeras cartas que envía Maraini a la estudiante son breves y prácticamente carentes de contenido, pero en esencia fundamental para despertar en el lector cierta antipatía hacia la autora por su desinterés y falta de empatía con las aspiraciones de la joven.

Ante la negativa de Dacia Maraini de hacerse cargo de la investigación sobre santa Clara, la estudiante insiste en otra carta en la que hace referencia a las palabras de la autora en una entrevista en la que habla sobre el proceso previo a tomar la decisión de embarcarse en la escritura de una historia. Se trata de la respuesta de Maraini a una pregunta que se le plantea muy a menudo, una de las últimas veces en una entrevista concedida a Domani Press News en 2017: 
Lei è una delle scrittrici italiane più amate e tradotte anche all'estero...cosa rappresenta per lei la letteratura? Come nasce la scrittura di un romanzo, si parte da una esigenza pedagogica o è un puro flusso di coscienza alla Joyce?

Nasce da un personaggio che viene a bussare alla mia porta. Io apro, offro un thè con dei biscotti. Il personaggio mi racconta la sua storia e poi di solito se ne va. Ma quando, dopo avere bevuto il thè e mangiato i biscotti, dopo avermi raccontato in breve la sua storia, mi chiede anche la cena e poi un letto per dormire, e la mattina dopo anche un caffè col latte, vuol dire che si è accampato nella mia testa e vuole rimanerci. È il momento in cui capisco che devo raccontare quella storia. (Maraini 2017:1)

La estudiante siciliana escribe a la autora que ella misma es una de esos personajes que llaman a su puerta, para impulsarla a escribir sobre santa Clara, y abre así una serie de cartas en las que cuenta características de su propia personalidad y de su vida en las que encuentra conexión con las de santa Clara. En una de ellas, Clara Mandalà introduce el concepto de corpo felice al cual aspira y que Maraini ha desarrollado con posterioridad, de forma más concreta en su última novela, ya mencionada, Corpo felice. Storia di donne, rivoluzioni e un figlio che se ne va (2018a). En la novela que nos ocupa, la autora deposita su propio pensamiento en la otra protagonista del intercambio epistolar en vez de en sí misma. De esta forma, aprovecha esta estructura literaria para mantener un diálogo consigo misma. Sin embargo, la estudiante siciliana se limita a afirmar en su carta «la mia aspirazione vera, glielo dico con la solita sincerità, è diventare un corpo felice» (Maraini 2013:16). En ningún momento explica explícitamente a qué se refiere. Preguntada Maraini en una reciente entrevista por el título de su última obra Corpo felice. Storia di donne, rivoluzioni e un figlio che se ne va (2018a) y el significado del mismo, la escritora explica que un cuerpo feliz es «un corpo capace di dare felicità ma anche di prenderla per sé» (Maraini en Kobo, 2018b). Tradicionalmente el cuerpo de las mujeres ha sido considerado un medio para dos fines: el de dar placer y el de dar vida. En el primer caso, históricamente ha sido nulo el interés y la reflexión sobre el placer sexual en las mujeres, a quienes se ha considerado seres pasivos y sin participación de ese placer. En el segundo caso, la historia de la humanidad se caracteriza por no haber dado a la capacidad de la mujer para crear vida el lugar de importancia crucial que en efecto tiene, y la cultura patriarcal ha logrado que esta capacidad sea percibida como un mero trámite, exento de importancia y de implicaciones en sí mismo. De nuevo encontramos en este punto la intención de Dacia Maraini de contribuir con su literatura al reconocimiento de la mujer como agente con un papel activo y protagonista en la sociedad. Así, podemos concluir que lo que la estudiante siciliana quiere decir cuando expone que su máxima aspiración es poseer un cuerpo feliz, es que su máxima aspiración es desprenderse de todos los lastres culturales y sociales que, por su condición femenina, sigue arrastrando en pleno siglo XXI.

La confirmación de esta tesis la encontramos en la siguiente carta que envía Clara Mandalà a Maraini. En esta ocasión la estudiante utiliza la carta para contarle a la escritora cómo es físicamente y cómo los estereotipos que la sociedad ha impuesto para 
las mujeres la han llevado a, por ejemplo, «cammino malamente su questi trampoli che si usano adesso e che io mi ostino a calzare ma mi stanno proprio male, me ne rendo conto» (Maraini 2013:17). La estudiante afirma en su carta que se siente perdida en este mundo y cree que descubriendo quién era santa Clara podrá descubrir algo más de sí misma. Así, comienza a exponer a Maraini todos los puntos de conexión que encuentra entre la santa y ella misma. El primer aspecto que destaca la estudiante es que santa Clara de Asís ayunaba, al igual que ella, y expone las circunstancias en las que se produce este ayuno:

Fino a questo momento ho pensato che il mio digiunare derivasse da odio verso il mio corpo. Ma perché poi si dovrebbe odiare il proprio corpo? Solo perché non corrisponde ai canoni di belleza più comuni? [...]. Sa cosa mi ha fatto smettere di rigettare tutto quello che mangiavo? Non i rimproveri di mia madre, non gli sguardi terrorizzati di mio padre [...]. Ma l'odore. Ho smesso perché l'odore del vomito mi saliva nelle narici e rimaneva lì tutto il giorno come una maledizione incancellabile [...]. Era l'odore della mia anima, mi capisce? L'odore di un corpo infelice che contibuava ad appartenermi nonostante l'avessi disconosciuto più volte.

Chiara digiunava. E dopo di lei hanno digiunato tante mistiche. Ho letto in un suo testo teatrale su santa Catarina da Siena che a trentatré anni, l'età della morte di Cristo, si è lasciata morire di fame [...].Comunque quello che le ha raccontato sulla santa mi ha fatto venire in mente che forse alla fine, nel rifiuto del cibo di molte donne e ragazze c'è una richiesta di spiritualità. (Maraini 2013: 19-21)

De nuevo en esta carta Dacia Maraini está depositando su propio pensamiento en la otra interlocutora, en la estudiante siciliana que, después de preguntarse por qué alguien tendría que odiar su propio cuerpo, dice llegar a la conclusión de que cuando las mujeres ayunan o vomitan los alimentos en la actualidad es un acto en busca de cierta espiritualidad. Esta reflexión la ha expresado en varias ocasiones la escritora, cuya opinión es que, al igual que «las místicas en la Edad Media rechazaban los alimentos terrenales para recibir mejor los espirituales, las mujeres que sufren de anorexia hoy en día están expresando un deseo inconsciente de espiritualidad en un mundo que ve el cuerpo femenino sólo como fuente de placer sexual y de decoración $»^{2}$. Sin embargo, la Dacia Maraini de la novela continúa mostrándose indiferente ante las reflexiones de Clara Mandalà y se sigue mostrando reacia en sus cartas ante la idea de llevar a cabo la investigación sobre santa Clara. Este recurso es utilizado por la autora para que la estudiante tenga que seguir recurriendo a la correspondencia para exponer otras coincidencias entre su vida y la de Clara de Asís, y así intentar involucrar a la escritora en la tarea. Por tanto, la relación entre ambas continúa siendo bastante fría e se percibe cierta desesperanza en el relato de Clara Mandalà, quien parece que escribe ya sólo por expresar lo que siente más que por tener esperanza en llegar a convencer a Maraini:

2 La cita procede de una entrevista concedida en febrero 2019 a María Belén Hernández González, para el artículo «Cibo e corpo femminile nella narrativa di Dacia Maraini» en curso de publicación. 


\section{Cara scrittrice}

Chiara era vergine. Io sono vergine. Non perché abbia fatto un voto di castità, o per ubbidire a una legge della Chiesa, ma per inappetenza sessuale e forse per pura noia. Il sesso mi sembra sgangherato e prevedibile. L'amore un sogno irraggiungibile.

Chiara invocava il silenzio. Ed io abito nel silenzio. Non riesco a parlare con nessuno. Le voci qui rimbalzano sulle pietre nere che ha lasciato la lava scorrendo e solidificandosi, scivolano lungo le nuove strade asfaltate, ma sono voci prive di significato. Non riesco a trovare qualcuno che parli con una voce significante. Per questo mi rivolgo agli scrittori. (Maraini 2013:22-23)

Encontramos en esta carta dos nuevos puntos de conexión entre Clara Mandalà y santa Clara, además del ayuno: el hecho de que ninguna mantiene relaciones sexuales y el papel del silencio en sus vidas. Obviamente en el caso de santa Clara la decisión de ser virgen es debida al voto de castidad, pero la Clara del siglo XXI relata otros motivos que la llevan a la misma determinación. Estos motivos dejan entrever el desencanto que la estudiante siente hacia el amor y las relaciones sexuales, lo percibe como algo desposeído de importancia y de emoción probablemente por cómo es tratado este asunto en la sociedad en la que vive, en la que todo es accesible, rápido y fácil. Probablemente la autora está de nuevo estableciendo un paralelismo entre el rechazo al sexo expresado por la estudiante con la decisión de santa Clara de dedicar su vida a Dios y no a un hombre, como estaba previsto para ella por su familia. Maraini estaría insinuando en este punto una posible motivación profunda común: la aspiración a la independencia afectiva, a la autonomía y, en última instancia, al logro de ese cuerpo feliz que hemos mencionado. Por otra parte, el silencio en la vida de santa Clara era buscado por ella misma para sentirse más cercana a Dios; la joven siciliana, sin embargo, se lamenta de vivir en el silencio por no encontrar a su alrededor voces con significado. Por ese motivo dice dirigirse a los escritores. A pesar de estos razonamientos, la Maraini de la novela sigue sin terminar de comprender las intenciones de Clara para dirigirse a ella, hecho que favorece que continúe el intercambio epistolar entre ellas:

\section{Cara Chiara}

Capisco, o tento de capire le sue ragioni. Ma non mi convincono. Cosa posso fare per lei? [...] (Maraini 2013: 23)

Después de recibir esta respuesta, Clara Mandalà decide no darse por vencida y continúa con la exposición de sus argumentos. De este modo, escribe que la pobreza es otra circunstancia que comparte con santa Clara (Maraini 2013: 25), si bien reconoce que la santa la eligió mientras que a ella se le impuso y, a pesar de ello, la aceptó sin verse atrapada por la necesidad de consumismo que se da habitualmente en los jóvenes de su edad. A partir de entonces y poco a poco en las cartas sucesivas que se intercambian Clara y Maraini, se puede detectar un progresivo acercamiento de la escritora a la situación de la estudiante y a su preocupación, sobre todo en el momento en que hablan sobre la pasión por la lectura e intercambian los conocimientos que una y otra han podido extraer de los libros sobre la Edad Media: 
Cara scrittrice

Lei ha ragione, forse io teatralizzo. Forse afferro la spada e la agito per aria, come se fossi una guerriera del Medioevo [...]. Intanto mi ingozzo di libri. Un libro tira l'altro. Sono peggio delle ciliegie. Ascolti: ho trovato il libro di cui mi ha accennato, Una solitudine abitata. Chiara d'Assisi di Chiara Frugoni [...]. Sono andata in biblioteca e ho trovato altri volumi che parlano della santa [...]. Sono tante, stanno tutti sul mio letto. Passo dall'uno all'altro con ingordigia. Eppure mi sembra che leggendo, invece si saperne di più, ne so di meno. Cosa sappiamo del Medioevo? [...] (Maraini 2013: 26-27)

\section{Cara Chiara}

Non mi mandi tutti quei libri per carità [...]. Inoltre la mia casa già gronda di libri [...]. Fra un po' dovrò uscire io per fare posto ai volumi. Sa che sono già quasi diecimila, e alcuni stanno in pila per terra perché non ho scaffali dove cacciarli? (Maraini 2013: 28)

\section{Cara scrittrice}

Le dico sinceramente che Chiara non può aspettare: ha preso il tè da lei, ma ora vuole un letto per dormire, insomma si è accampata nella casa della sua immaginazione e vuole essere ascoltata. La prego, non ci scacci. Tengo per mano una donna straordinaria che è stata troppo spesso dimenticata, trascurata, ignorata dalla memoria civile di questo Paese. Tutti quei libri che le ho citato sono pubblicati quasi interamente dalla benemerita Casa Porziuncola di Assisi [...]. (Maraini 2013: 29-30)

\section{Cara Chiara}

Ho ricevuto i suoi libri. Grazie. Ma li ho rimandati alla biblioteca. Le ho detto che non posso accontentarla. E inoltre, insisto: perché vuole che sia io a raccontare di Chiara? Fra l'altro il Medioevo mi è estraneo. O per lo meno lo conosco poco, quel tanto che mi è venuto dalla lettura di Huizinga [...]. Ma ora che ci penso ho letto anche un libro che mi ha molto incuriosito, sulle beghine, termine di per sé comico, con cui si prendevano in giro le donne troppo bigotte e corte di pensiero [...]. Ecco, mi piacerebbe scrivere un libro sulle beghine, anzi su una beghina [...]. Grazie, Chiara, per avermi riavvicinata al Medioevo e avere trovato nelle sue pieghe una creatura serena, inteligente e generosa come la beghina. (Maraini 2013: 30-31)

Desde este momento, la Maraini de la novela reconoce y acepta que Clara Mandalà ha conseguido que se despierte en ella el interés por la época y por recuperar los conocimientos que ha adquirido sobre ella a lo largo de su vida, hecho que funciona como paso previo a que la escritora se interese también por descubrir la figura de santa Clara. Se abre así el último periodo de intercambio de cartas entre Maraini y la estudiante, en las que mantienen conversaciones basadas en sus reflexiones sobre la importancia de la virginidad, el significado de la santidad y de la laicidad, el tipo de milagros que sue- 
len atribuirse a las santas en sus contextos... y en pocas páginas ambas se encuentran inmersas en una puesta en común de los conocimientos sobre la vida de santa Clara que cada una va descubriendo a partir de diversas lecturas. De este modo, se puede dar por demostrado uno de los objetivos de Dacia Maraini cuando se decantó por una propuesta epistolar para la apertura de esta novela: hacer ver la necesidad de que las mujeres en determinadas posiciones se responsabilicen y tomen de la mano a mujeres en dificultades. La escritora en este caso propone una actitud en ella misma en la novela de tutora o guía para Clara Mandalà en el descubrimiento de la figura de la santa como referente histórico feminista. En efecto, no es un hecho casual que la autora elija a una estudiante de diecinueve años como la persona que le pide ayuda para resolver un problema existencial. Como se puede deducir a partir de la lectura de las cartas de la joven siciliana, ella no es alguien con una personalidad definida y las ideas claras sino una persona inmersa en un mar de dudas. Así, el papel de Maraini en la novela es el de aportarle algo de estabilidad desde afuera, al prestarle ayuda con la empresa que la tiene casi obsesionada. En las últimas cartas de esta primera parte que la estudiante envía a Maraini su tono es mucho más relajado, ha conseguido la ayuda que esperaba de la escritora y siente que puede estar tranquila:

\section{Cara scrittrice}

Ecco, lei è già entrata nella storia. Ha già afferrato la piccola magrissima mano di monna Chiara, figlia di Favarone di Offreduccio e di Ortolana Fiumi. Decida lei como continuare. Le auguro buon viaggio. Alla fine del viaggio le chiederò solo di testimoniare in mio favore. Conoscendo meglio Chiara, la mia omonima, conoscerò me e il mio destino e forse, spero, qualcosa del mio stare al mondo che è un enigma. Vorrei imparare a smettere di digiunare ma questo succederà solo se arriverò a possedere un corpo felice. Lei avanza l'ipotesi che il corpo di Chiara non fosse felice, nonostante la felicità estrema dell'estasi spirituale. Da cosa lo deduce? Dalla malattia? [...]. (Maraini 2013:51)

\section{Cara Chiara}

Certo, con gli occhi di oggi sembra quasi una follia, l'accanimento di una volontà ferrigna contro un giovane corpo di donna. Quel cercare con determinazione la mortificazione, il sacrificio. Ma quanto di questa cocciuta umiliazione del corpo apparteneva all'epoca e quanto a lei, a quella ragazzina testarda e determinata? [...].

Certo non rimaneva molto spazio per pensare a se stessi o al proprio corpo quando le regole del convento venivano rispettate. Le monache dovevano alzarsi alle sei. $\mathrm{E}$ recitare la Prima. Dopodiché potevano fare una piccola colazione con pane e acqua. Seguivano tutti i lavori per mantenere pulito il convento [...].

Possiamo imaginarla la nostra Chiara dai piedi callosi e le mani rovinate dai lavori domestici, che non perde mai la calma, anche di fronte alle difficoltà delle malate, con cui si mostra indulgente, accudente [...].(Maraini 2013:52-58) 
Cara scrittrice

Lei ormai ne sa più di me. Sono contenta che Chiara l'abbia decisamente afferrata per la mano e ora la stia tirando dalla sua parte. Sono io che barcollo [...]. Vorrei smettere di sognare. Ma forse no, forse riprenderò a digiunare, tanto da lasciare sul letto la mia spoglia come un bruco ansioso di trasformazione, per tirare fuori le ali sgualcite e prendere faticosamente il volo. (Maraini 2013: 58-59)

Esta es la última carta que la escritora recibe en la apertura de la novela de Clara Mandalà. Podemos deducir que, una vez ha conseguido que Maraini se embarque en la aventura de investigar y redescubrir a santa Clara de Asís, la estudiante siente que ha cumplido con su misión y se queda tranquila. A partir de entonces comienza la segunda parte de la novela, una mezcla entre diario y novela histórica sobre las circunstancias en las que vivió y se desarrolló la figura de la santa, con continuas aportaciones ensayísticas de la autora cuando reflexiona sobre esa realidad, entremezcladas con la presencia de testimonios originales del proceso de canonización de Clara de Asís. Maraini también incluye aquí una recopilación de escritos de los padres de la Iglesia que ponen en duda el valor de la mujer, porque la autora pretende que este hecho se tenga presente hoy en día y se destaque y aprecie el papel de mujeres como santa Clara en la lucha contra esos prejuicios. De esta manera, se puede concluir que el fragmento epistolar del principio de la novela es un pretexto para atrapar a los lectores e involucrarles en la historia, con el objetivo de conseguir el fin último de la novela: la recuperación de una figura histórica femenina que ha sido olvidada, desprestigiada y marginada por la posteridad. Como tantas otras. Y para eso escribe Dacia Maraini, para devolverlas a la vida y conseguir que por fin tengan el lugar que por méritos propios les corresponde. Además, la recuperación del formato epistolar para cerrar la novela tras el fragmento ensayístico central confirma la estrategia de Maraini para crear una novela moderna y actual como gancho que atraiga al lector. Así, la escritora cumple con su mencionado objetivo fundamental, el de rescatar para el conocimiento de los lectores de hoy en día a mujeres que a lo largo de la historia han luchado por la libertad de su sexo en un mundo que las denostaba. De hecho las últimas cartas que encontramos en la parte final de la novela, entre las que de nuevo Maraini intercala un breve fragmento de su diario con reflexiones sobre la santa, nos muestran a una Clara Mandalà que se siente liberada gracias al escrito que la autora ha llevado a cabo sobre santa Clara. Después de meses sin dar señales de vida, la joven se muestra agradecida a Maraini por haber respondido a su petición y afirma haber encontrado su camino, el de convertirse en clarisa:

\section{Cara scrittrice}

Io non credo alle verità del mondo. Ma a quelle della fede credo. Overo credo al mistero che avvolge tutte le cose. Per quello mi piace l'idea di chiudermi in convento. Anche se oggi suona quasi un anacronismo, anche se i miei mi danno della pazza, anche se c'è una parte di me che protesta. Ma ho sentito la voce di Chiara [...]. È struggente quello che sento. Non so se si tratti di una vera vocazione. Ma da quando ho deciso di rinchiudermi, sono passate tutte le mie paure, i miei dubbi, le mie inquietudini. Forse non mi conoscevo [...]. (Maraini 2013: 218-219) 
En estos últimos intercambios de correspondencia la Maraini de la novela vuelve a mostrarse hostil con la estudiante debido a que ella le confiesa que ha podido acceder al escrito sobre santa Clara entrando sin autorización en su ordenador, y porque la estudiante mintió en alguno de los datos que dio sobre sí misma. Este enfrentamiento inicial vuelve a dar paso poco a poco, como ocurre en la primera parte descrita, a un intercambio de opiniones e información sobre determinados aspectos de la investigación sobre la santa que han suscitado dudas en una o en la otra. Aquí Maraini repite la estrategia de la aparente desgana o enfado inicial como excusa para que se establezca la comunicación entre las dos y dar pie así al intercambio de impresiones sobre la figura de Clara de Asís. Así, reflexionan sobre el significado de la palabra sororidad, en italiano «sororità» (Maraini 2013: 223-224); el desprecio que santa Clara sentía hacia su cuerpo, motivado según Mandalà por la «dimenticanza di sé [...], la pratica del servire gli altri, la gioia del donarsi» (Maraini 2013: 229-230); o la vocación de la estudiante de convertirse en monja, sobre la que Maraini le escribe:

\section{Cara Chiara}

Da quando ha deciso di entrare in convento ha lasciato le sue sfide, le sue inquietudini. Non so se questo sia meglio o peggio per lei. Per me è una perdita. Non riesco a concepire un pensiero imbrigliato e non capisco come oggi ci si possa chiudere dietro una grata se non per un atto di crudeltà verso se stessi. (Maraini 2013:233)

Mandalà responde a esta carta trasmitiendo de nuevo a su destinataria que ella es ahora una persona diferente a la que comenzó a escribir, que su vocación «non è una scelta volontaria. Non segue un computo di perdite e guadagni» (Maraini 2013: 233). Verificamos que todo el proceso de dudas por el que la estudiante estaba atravesando, efectivamente precisaba de ayuda externa, en este caso de la escritora, para hallar una solución. Tras leer el escrito de Maraini sobre santa Clara, la joven se siente despojada de toda duda y toma la decisión firme de entrar en el convento, a pesar de ser consciente de que ni ella ni su entorno tienen del todo claro que sea lo mejor. El hecho es que Maraini ha logrado el objetivo de mostrar la necesidad de que mujeres como ella, maduras y en determinadas posiciones de privilegio, atiendan a las necesidades y las dudas de las jóvenes que a menudo se encuentran desorientadas y en busca de un sitio en esta sociedad lleno de lastres para las mujeres. La novela concluye con una carta de la estudiante en la que expresa su gratitud:

\section{Cara scrittrice}

Non so come esprimerle la mia gratitudine. So che ha faticato tanto. Spero che non si sia ammalata e che le sue costole siano sane nonostante i dolori. Mi sento un poco in colpa per averla tirata i questa vicenda ma spero ne sia valsa la pena. Io mi sono innamorata di Chiara, tanto da seguirla in convento. E di questo la ringrazio. Credo di avere capito solo ora cosa significhi un corpo felice. Non mi stringerò il petto con ua corda fatta di peli di coda di cavallo, né mi legherò alla vita un corpetto foderato di pelle di porco. Ma credo che canterò in Chiesa, dove finalmente la mia voce potrà aprire le sue ali [...]. (Maraini 2013: 249) 
Así pues, a partir del análisis desarrollado sobre el empleo del género epistolar en la narrativa de Dacia Maraini, podemos determinar que la autora recurre a él con frecuencia porque confía en su efectividad para acercar lo máximo posible las vivencias de los personajes del relato a los lectores. Probablemente debido a que la autora es consciente de que cuando una persona se expresa por escrito, lo hace más concienzuda y reflexivamente que cuando habla. De este modo encontramos numerosas escritoras de cartas en sus novelas, desde Bianca en Lettere a Marina (1981), pasando por Marianna en la aplaudida La lunga vita di Marianna Ucría (1990) o Vera en Dolce per sé (1997), y hasta llegar a la propia Dacia Maraini como personaje presente en su última novela, publicada el año pasado, en la que escribe una carta a su hijo perdido.

Por otra parte, en cuanto a los objetivos perseguidos por la autora con el planteamiento de su novela Chiara d'Assisi. Elogio della disobbedienza (2013) podemos destacar algunas conclusiones. En primer lugar el por qué de la estructura de la novela, que se revela moderna y atractiva al contar con dos formatos bien diferenciados e intercalados oportunamente para mantener atrapado el interés del lector: las páginas de intercambio epistolar entre la estudiante siciliana y Maraini que abren y cierran la novela, y las destinadas a reflejar el diario de la autora, en las que lleva a cabo su ensayo sobre la figura histórica de santa Clara de Asís con la presencia de fuentes originales sobre su proceso de canonización. En segundo lugar, la introducción a lo largo de todo el relato de una serie de ideas fundamentales en la vida y en la obra de la autora: el interés que despiertan en ella las figuras femeninas religiosas que a lo largo de la historia han actuado desde su posición y circunstancias por la liberación de la mujer, como santa Catalina de Siena o nuestra Clara de Asís; la opinión de Maraini sobre el papel y la actitud de la mujer en la actualidad, con la presentación de la estudiante Clara Mandalà como una joven llena de dudas e inquietud por no encontrar su lugar en el mundo y sentirse atrapada por los prejuicios y contradicciones sociales; y en relación con la situación de Clara Mandalà, la introducción de conceptos frecuentes en el discurso de Maraini como el de corpo felice que hemos analizado en el artículo, y la necesidad que defiende la escritora del ejercicio por parte de mujeres en determinadas posiciones de tutoras o mentoras de otras en circunstancias más desfavorecidas. Para concluir, debemos destacar la evolución del tono en la relación entre la estudiante y Maraini en la novela, que pasa de ser frío en las primeras cartas a entusiasta en las que ambas intercambian opiniones sobre conceptos estrecha e históricamente unidos a la realidad femenina, tales como la importancia de la virginidad, el rechazo a los alimentos o el silencio.

\section{BIBLIOGRAFÍA}

ARRIAGA, Mercedes (2006): Desde Andalucía: mujeres del Mediterráneo. Sevilla: Arcibel.

GINZBURG, Natalia (1984): La città e la casa. Turín: Einaudi.

MARAINI, Dacia (1981): Lettere a Marina. Milán: Bompiani.

(1990): La lunga vita di Marianna Ucrìa. Milán: Rizzoli.

(1996): Un clandestino a bordo. Milán: Rizzoli. 
(1997): Dolce per sé. Milán: Rizzoli.

(2003): «La lunga vita di santa Caterina raccontata da Dacia Maraini», Rossana Campisi, La Repubblica.it,13/03/2003. [https://ricerca.repubblica.it/repubblica/archivio/repubblica/2003/03/13/la-lunga-vita-di-santa-caterina-raccontata.html; 03/04/19]

(2008): Il treno dell’ultima notte. Milán: Rizzoli.

(2013): Chiara d'Assisi. Elogio della disobbedienza. Milán: Rizzoli.

(2017): «Intervista - Dacia Maraini: "Fare progetti per il futuro tutti insieme è la forma più saggia per affrontare il domani"», Simone Intermite, Domani Press News, 20/07/2017. [https://www.domanipress.it/dacia-maraini-fare-progetti-per-il-futuro-tutti-insieme-e-la-forma-piu-saggia-per-affrontare-il-domani/; 26/03/19]

(2018a): Corpo felice. Storia di donne, rivoluzioni e un figlio che se ne va. Milán: Rizzoli.

(2018b): «Il corpo felice delle donne. Intervista a Dacia Maraini», Rosa Carnevale, Blog Kobo, 17/12/2018. [https://www.kobo.com/it/blog/il-corpo-felice-delle-donne-intervista-a-dacia-maraini; 02/04/2019]

(2019a): «Dacia Maraini, le parole di una vita: "Un corpo felice è quello che desidera, sogna, dà e riceve"», Lavinia Farnese, Vanity Fair, 14/01/2019. [https://www.vanityfair.it/show/libri/2019/01/14/dacia-maraini-corpo-felice-anni-parole-scrittrice-foto; 01/04/2019]

(2019b): «Cibo e corpo femminile nella narrativa di Dacia Maraini», María Belén Hernández, en curso de publicación.

RODRÍGUEZ Fierro, Mercedes (1997): «La novela epistolar en Italia y España: el drama y la estrategia de la ocultación desde Galdós a Natalia Ginzburg», Cuadernos de Filología Italiana. Volumen 4: 203-215. Madrid: Servicio de Publicaciones UCM.

SOLORZA, Paola Susana (2015): «El feminismo italiano y español de la década de 1980. Perspectivas sociales y representaciones literarias: Lettere a Marina (1981) de Dacia Maraini y Para no volver (1985) de Esther Tusquets», Cuadernos Intercambio sobre Centroamérica y el Caribe. Volumen 12: 129-152. San José: Universidad de Costa Rica.

STASIO, Loreta de (2006): «El teatro y la escritura femenina contemporánea: Recorrido, analogías y contrastes de dos dramaturgas italianas», Mercedes Arriaga (ed.). Desde Andalucía: mujeres del Mediterráneo. Sevilla: Arcibel Editores, 136-149. 


\section{PERFIL ACADÉMICO Y PROFESIONAL}

Almudena Miralles Guardiola es licenciada en Traducción e Interpretación por la Universidad de Murcia (2010), con Máster en Traducción Editorial (2011) y Máster en Formación del Profesorado (2013) en la misma universidad. Actualmente realiza estudios de postgrado dentro del programa de doctorado de Artes y Humanidades de Eidum con un proyecto tesis sobre la obra de Dacia Maraini.

Ha colaborado como traductora en conferencias en la Universidad de Murcia y participó en la traducción al español del libro Confines lógicos de la matemática (2011) de Giuseppe Raguní. Ha trabajado como profesora de italiano y francés en distintas asociaciones y empresas. Actualmente es profesora de Enseñanza Secundaria.

Sus intereses en investigación se centran en estudios de producciones literarias con perspectiva de género.

Fecha de recepción: 21/04/2019

Fecha de aceptación: 26/05/2019 